\title{
Cross-linking e segmento de anel corneano intraestromal
}

\section{Cross-linking and intrastromal corneal ring segment}

\author{
Adimara da Candelaria Renesto ${ }^{1}$, Marta Sartori ${ }^{1}$, Mauro Campos $^{1}$
}

\section{RESUMO}

O cross-linking corneano é um procedimento usado para a estabilização mecânica e aumento da rigidez corneana em pacientes com ceratocone (reduzindo a possibilidade de progressão), e também em processos inflamatórios de afinamento corneano. Os segmentos de anéis corneanos intraestromais têm como princípio o aplanamento central da córnea. Inicialmente utilizados para correção de baixa miopia, a principal indicação atual é em pacientes com ceratocone, para melhorar a acuidade visual não corrigida, a acuidade visual corrigida e permitir uma melhor tolerância ao uso de lentes de contato como também retardar a necessidade de um transplante de córnea. O objetivo deste artigo é revisar algumas publicações relacionadas ao crosslinking corneano e à inserção do segmento de anel intraestromal, apresentando suas indicações, resultados e complicações relatadas até o momento.

Descritores: Córnea; Riboflavina; Colágeno; Terapia ultravioleta; Ceratocone

\begin{abstract}
Corneal cross-linking is a procedure used for stabilizing the cornea in patients with progressive keratoconus by increasing corneal rigidity, and it is also used in corneal inflammatory melting process. The intrastromal corneal ring segments act by flatting the center of the cornea. Originally designed for the correction of mild myopia, the segments are now being used for reduction of keratoconus in order to improve the uncorrected visual acuity, the best spectacle corrected visual acuity, to allow good tolerance to the use of contact lenses and delay the need for corneal grafting procedures. The present text presents a review of corneal cross-linking and insertion of intrastromal corneal ring segments, emphasizing their indications, results and complications related until now.
\end{abstract}

Keywords: Cornea; riboflavin; collagen; Ultraviolet therapy; keratoconus

\section{HISTÓRICO}

A investigação biomecânica de córneas humanas ectásicas iniciou-se a partir de 1980 revelando diferenças significantes na sua elasticidade comparada com córneas normais, indicando uma diminuição na rigidez corneana em córneas com ceratocone ${ }^{(1)}$. A base microscópica destes achados ainda é desconhecida, mas acredita-se em uma redução das ligações cruzadas de colágeno corneano e também das ligações moleculares entre os proteoglicanos corneanos estromais ${ }^{(2)}$.

Até 1998, nenhum tratamento conservador para ectasia corneana estava disponível. O princípio do cross-linking corneano (CXL) pela combinação da irradiação ultravioleta A (UVA) e o fotossintetizador riboflavina é o aumento da rigidez corneana devido ao aumento das ligações intrafibrilares e interfibrilares entre as moléculas de colágeno da córnea levando à diminuição da progressão de ectasias corneanas ${ }^{(3)}$. Os primeiros estudos de CXL em olhos humanos iniciaram-se em 1998 na Universidade de Dresden, Alemanha por Wollensak, Spoerl e Seiler(4).

Trabalho realizado no Departamento de Oftalmologia, Setor de Cirurgia Refrativa, Universidade Federal de São Paulo - UNIFESP - São Paulo (SP), Brasil.

${ }^{1}$ Médicos, Departamento de Oftalmologia, Setor de Cirurgia Refrativa, Universidade Federal de São Paulo - UNIFESP - São Paulo (SP), Brasil.

Endereço para correspondência: Adimara da Candelaria Renesto. Rua Carlos Sampaio, 94 - Apto. 22 - São Paulo (SP) - CEP 01333-020

E-mail: adimararenesto@uol.com.br

Os autores não apresentam qualquer tipo de interesse comercial nos produtos ou equipamentos mencionados no texto.

Recebido para publicação em 14.05.2010

Última versão recebida em 07.11.2010

Aprovação em 08.11.2010

Nota Editorial: Depois de concluída a análise do artigo sob sigilo editorial e com a anuência dos Drs. Sérgio Kwitko e Adriana dos Santos Forseto sobre a divulgação de seus nomes como revisores, agradecemos suas participações neste processo.

\section{INTRODUÇÃO}

O ceratocone é uma doença relativamente frequente com uma incidência de 1 em 2.000 na população geral, afetando jovens pacientes ${ }^{(5)}$. Ao redor de $21 \%$ dos pacientes com ceratocone serão submetidos ao transplante de córnea ${ }^{(5)}$. Métodos atuais de tratamento, como os segmentos de anéis intracorneanos, ceratectomia fotorrefrativa e o uso de lentes de contato apenas corrigem o erro refrativo não impedindo a progressão da doença ${ }^{(5)}$. Pela primeira vez, um novo tratamento baseado nas ligações cruzadas de colágeno com a ajuda da irradiação ultravioleta A e o fotossintetizador riboflavina foi introduzido. Este tratamento tem como objetivo interferir no colágeno corneano de pacientes com ceratocone e mudar as propriedades biomecânicas intrínsecas deste colágeno, impedindo assim a progressão da doença $a^{(5)}$.

\section{PrincípIo básico}

O CXL é um método largamente empregado na indústria de polímeros para o endurecimento de materiais e também em bioengenharia para a estabilização de $\operatorname{tecidos}^{(5)}$.

Usando UVA em um comprimento de onda de $370 \mathrm{~nm}$ e o fotossintetizador riboflavina (Figuras 1 e 2), esta é excitada em seu estado triplo produzindo espécies reativas de oxigênio, principalmente oxigênio livre e em menor quantidade radicais de ânion superóxidos. O oxigênio livre reage com várias moléculas induzindo ligações químicas covalentes e pontes entre grupos aminos das fibrilas de colágeno corneano (reação fotoquímica tipo II). O comprimento de onda de $370 \mathrm{~nm}$ foi escolhido porque é onde se dá o pico de absorção da riboflavina ${ }^{(5)}$. A riboflavina, vitamina B2 (peso molecular de $\left.376,37 \mathrm{~g} / \mathrm{mo}^{(6)}\right)$, é um fotossintetizador não tóxico, solúvel em água e penetra facilmente no estroma corneano na ausência de epitélio ${ }^{(3)}$.

\section{TÉCNICA}

A técnica padrão para o procedimento do $C X L$ corneano é a seguinte ${ }^{(6)}$ : Início do procedimento em sala cirúrgica em condições 


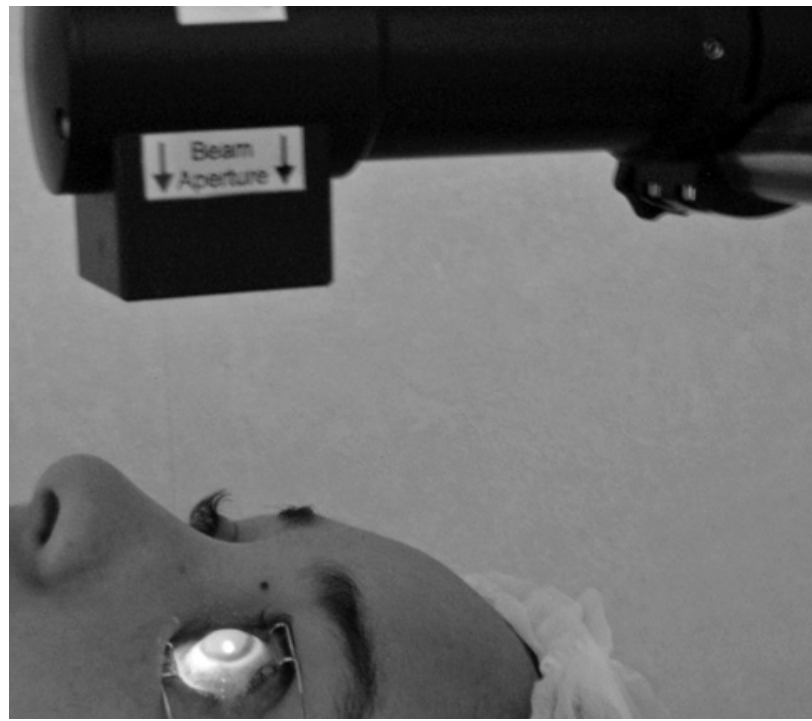

Figura 1. Procedimento do cross-linking corneano.

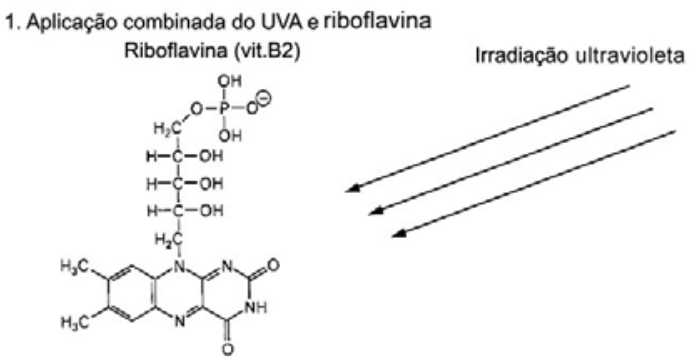

2. Produção de radicais de oxigênio

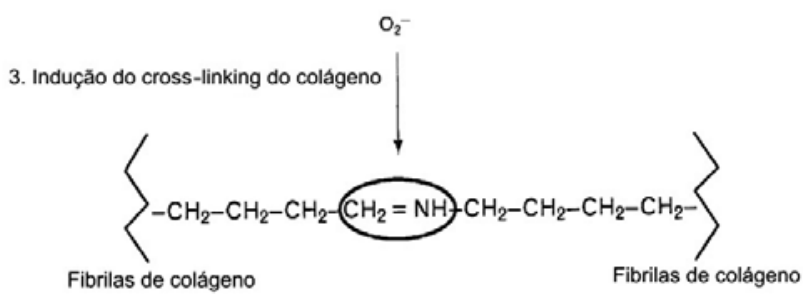

Figura 2. O princípio do cross-linking fotodinâmico pelo ultravioleta A e o fotossintetizador riboflavina.

de esterilização adequadas. Instilação de colírio de cloridrato de proximetacaína 0,5\% para anestesia local. Remoção do epitélio corneano central em $9 \mathrm{~mm}$ com o uso de uma espátula romba. Um fotossintetizador, colírio de solução de riboflavina 0,1\% (10 mg de riboflavina-5-fosfato em solução de $10 \mathrm{ml}$ de dextran-T-500 20\%), é aplicado na córnea a cada 5 minutos por um período de 30 minutos antes do início da irradiação UVA. Usando-se a lâmpada de fenda em luz azul de cobalto, o cirurgião confirma a presença da riboflavina na câmara anterior. A córnea então é exposta (uso de blefarostato) à irradiação de luz ultravioleta através de um aparelho em sólido estado, estando disponíveis para uso: O (UV-X System; Peschke Meditrade GmbH, Hunenberg, Switzerland), o X-Link (Opto Eletronica, São Carlos, Brazil(7)), e o CSO Vega CBM X linker (CSO, Florence, Italy $\left.{ }^{(8)}\right)$, que emitem luz em um comprimento de onda de $370 \pm 5 \mathrm{~nm}$ a uma irradiância de $3 \mathrm{~mW} / \mathrm{cm}^{2}$ ou 5,4 J/cm². A córnea é exposta por 30 minutos, onde a solução de riboflavina é aplicada a cada 5 minutos. Durante o procedimento, o cirurgião controla a centralização do tratamento pedindo ao paciente para manter o olhar em direção à sonda de emissão de luz. Após o tratamento, é colocada uma lente de contato terapêutica que é mantida até a completa reepitelização corneana. Uma combinação de colírio de antibiótico e anti-inflamatório hormonal é prescrita em média por 15 dias ou enquanto houver defeito epitelial. Podese utilizar também analgésicos orais em pacientes com queixa exacerbada de dor.

\section{EstudOS LABORATORIAIS}

Estudos têm sido conduzidos em relação aos efeitos biomecânicos, efeitos termomecânicos, efeitos morfológicos, efeitos histológicos, localização anterior do efeito principal e efeitos na resistência à colagenase.

\section{Efeitos biomeCÂNICos}

Usando um equipamento que avalia medidas de tensão-deformação em córneas porcinas e humanas, Wollensak et al. ${ }^{(9)}$ verificaram que houve um aumento significante da rigidez corneana após o CXL, indicado por um aumento de tensão em córneas porcinas de 71,9\% e em córneas humanas de 328,9\%. O efeito máximo do CXL encontra-se na porção de $300 \mu \mathrm{m}$ anterior da córnea. Assim, o maior efeito biomecânico em córneas humanas é explicado pela grande quantidade de estroma submetido ao efeito do CXL, visto que a espessura corneana humana é em média de $550 \mu \mathrm{m}$ comparada a $850 \mu \mathrm{m}$ de córneas porcinas.

\section{Efeitos termomecÂNICOS}

Em experimentos termomecânicos com córneas porcinas, o máximo encolhimento hidrotermal foi $70^{\circ} \mathrm{C}$ para controles não tratados, $75^{\circ} \mathrm{C}$ para córneas tratadas com UVA e riboflavina e $90^{\circ} \mathrm{C}$ para córneas tratadas com gluteraldeído, demonstrando a dependência da temperatura de encolhimento no efeito de CXL. Além disso, em córneas tratadas com UVA e riboflavina uma alta temperatura de encolhimento foi observada na porção anterior da córnea $\left(75^{\circ} \mathrm{C}\right)$ comparada à porção posterior do estroma $\left(70^{\circ} \mathrm{C}\right)$ devido ao alto grau de CXL no estroma anterior. A localização anterior do efeito do CXL é vantajoso para o endotélio corneano e para a preservação da curvatura anterior da córnea ${ }^{(10)}$.

\section{EFEITOS MORFológicos}

No estroma anterior de córneas de coelhos tratadas com UVA e riboflavina, o diâmetro das fibras colágenas estava significativamente aumentado em 12,2\% (3,96 nm), e no estroma posterior de $4,6 \%(1,63 \mathrm{~nm})$. O efeito do CXL é maior na metade anterior do estroma devido à rápida diminuição da irradiação UVA através do estroma corneano como resultado do aumento da absorção de UVA pela riboflavina ${ }^{(11)}$.

Bottós et al. ${ }^{(7)}$, em um estudo usando imunofluorescência confocal em córneas porcinas submetidas ao tratamento de CXL e possíveis variações de técnica, observaram uma zona de fluorescência anterior $(182,5 \pm 22,5 \mu \mathrm{m})$ altamente organizada e com uma linha de demarcação de fibras de colágeno apenas nos olhos com o tratamento clássico, o que não foi observado em outros grupos (grupo controle apenas com desepitelização da córnea; cross-linking sem desepitelização, ou desepitelização com aplicação isolada de riboflavina ou UVA), ressaltando a importância da remoção do epitélio corneano e da associação riboflavina-UVA para o efeito do tratamento de CXL.

Em um estudo de microscopia confocal a laser in vivo, Mazzotta et al. ${ }^{(12)}$, em um seguimento de três anos de pacientes submetidos ao $C X L$, demonstraram que não houve dano à região corneana limbar, a anatomia do plexo nervoso estava normal após um ano do procedimento e houve um aumento da densidade da matriz extra- 
celular no pós-operatório tardio pelo CXL do colágeno, evidenciado por uma linha de demarcação tardia a $340 \mu \mathrm{m}$ de profundidade.

\section{Efeitos histológicos}

Em coelhos tratados com riboflavina e irradiação UVA variando de 0,75 a $4 \mathrm{~mW} / \mathrm{cm}^{2}$, a apoptose de ceratócitos foi encontrada em profundidades variadas já com um dia de pós-operatório dependendo da irradiação utilizada. Com a irradiação padrão de $3 \mathrm{~mW} /$ $\mathrm{cm}^{2}$ a apoptose de ceratócitos estava presente a uma profundidade de até $300 \mu \mathrm{m}$. Um nível de citotoxicidade de irradiação UVA para os ceratócitos foi mostrado por volta de $0,5 \mathrm{~mW} / \mathrm{cm}^{2}$, correspondendo a doses de UVA de 0,86-1,39 J/. $\mathrm{cm}^{2(13)}$.

\section{LOCALIZAÇÃO ANTERIOR DO EFEITO PRINCIPAL}

Há vários achados que apontam para a localização anterior do efeito cross-linking na córnea. O diâmetro das fibras de colágeno está significativamente aumentado somente na metade anterior do estroma ${ }^{(10)}$. Em um estudo em que córneas porcinas e de olhos humanos doadores submetidas ao CXL foram cortadas com um microcerátomo a uma profundidade de $200 \mu \mathrm{m}$, em córneas porcinas o efeito de rigidez foi maior no flap anterior tratado $(261,7 \pm$ $\left.133,2 \times 10^{3} \mathrm{~N} / \mathrm{m}^{2}\right)$ em relação ao flap posterior tratado $(105,0 \pm 55,8$ $\times 10^{3} \mathrm{~N} / \mathrm{m}^{2}$ ) e flaps controles ${ }^{(14)}$. Um efeito semelhante foi observado em córneas humanas. Este estudo demonstrou que o tratamento da córnea com riboflavina e UVA aumenta significativamente a rigidez corneana até $200 \mu \mathrm{m}$. Isto é explicado porque 65\% de $70 \%$ da irradiação UVA foi absorvida dentro de $200 \mu \mathrm{m}$ e somente 20\% após $200 \mu \mathrm{m}^{(14)}$.

Todos esses achados mostram uma preservação do endotélio corneano e de estruturas oculares mais profundas.

\section{EFEITOS NA RESISTÊNCIA À DIGESTÃo ENZIMÁTICA}

O CXL do colágeno corneano pela riboflavina e UVA aumenta significativamente a resistência à digestão pela colagenase, pepsina e tripsina, especialmente na metade anterior da córnea. Botões corneanos porcinos tratados com o CXL, foram dissolvidos somente 13 e 14 dias após digestão de pepsina e colagenase respectivamente versus 6 dias de córneas controles não tratadas. A digestão pela tripsina foi observada no $5^{\circ}$ dia em córneas tratadas versus 2 dias em córneas controles. Esses achados encorajam o uso deste procedimento para o tratamento de úlceras corneanas ${ }^{(15)}$.

\section{Estudos CLínicos}

O primeiro estudo clínico no tratamento de cross-linking foi realizado por Wollensak et al. ${ }^{(4)}$. Neste estudo, com um tempo de seguimento de 3 meses a 4 anos, 22 pacientes com ceratocone progressivo foram submetidos ao CXL. Em todos os olhos tratados não houve progressão do ceratocone. Em 15 pacientes houve uma melhora da acuidade visual corrigida em 1,26 linhas de Snellen. Em 16 pacientes houve uma regressão e aplanamento do ceratocone em 2,01 dioptrias (D). Não houveram complicações, córnea e cristalino permaneceram transparentes, sem perda de células endoteliais ou alteração na pressão intraocular.

A tabela 1 mostra alguns resultados recentes da literatura no tratamento de cross-linking para ceratocone e outras ectasias.

\section{Rıscos e COMPLicações}

A irradiação ultravioleta geralmente representa um potencial perigo para o olho humano. Sabemos que a irradiação ultravioleta B (UVB 290-320 nm) é a principal responsável pelas queimaduras oculares causadas pelo sol ou fotoceratites.

No tratamento de cross-linking, apenas um pico de UVA (370 nm) é usado. Além disso, devido à proteção da riboflavina, todas as estruturas atrás do estroma corneano, incluindo endotélio corneano, câmara anterior, íris, cristalino e retina são expostas a uma radiação residual de ultravioleta que é menor do que $1 \mathrm{~J} / \mathrm{cm}^{2}$. Este valor é considerado seguro de acordo com as pesquisas da literatu$r^{(6-13)}$. Em um estudo de córneas porcinas, o nível de citotoxicidade para o endotélio corneano foi de $0,36 \mathrm{~mW} / \mathrm{cm}^{2}$, que poderia ser alcançado em córneas humanas com uma espessura estromal menor do que $400 \mu \mathrm{m}^{(19)}$. Assim, é de fundamental importância a realização da paquimetria pré-operatória para o procedimento de crosslinking, para a exclusão de córneas com menos de $400 \mu \mathrm{m}$ estromais, e também checar a irradiância UVA usando o calibrador do equipamento de CXL.

Em relação às complicações do CXL, Koller et al. ${ }^{(20)}$ avaliaram 117 olhos de 99 pacientes com ectasia primária, em um ano de seguimento pós CXL. Neste estudo avaliou-se a razão de complicação do CXL e recomendações para evitá-la. A porcentagem de olhos com perda de 2 ou mais linhas de Snellen foi 2,9\%. A razão de falha do CXL (olhos com progressão da ectasia) foi de 7,6\%. Idade acima de 35 anos e acuidade visual corrigida pré-operatória meIhor do que 20/25 foram identificados como fator de risco para complicações. Infiltrados estéreis foram observados em 7,6\% dos casos e cicatriz estromal em 2,8\%. Os autores concluíram que algumas mudanças no critério de inclusão para o CXL podem reduzir falhas no tratamento e complicações.

Há também relato de caso relacionando o procedimento do CXL a algumas complicações como ceratite lamelar difusa após o CXL em paciente com ectasia corneana pós laser in situ keratomileusis(21).

Outras potenciais complicações incluem ceratite infecciosa, "haze" estromal e defeito epitelial persistente.

Tabela 1. Resultados do tratamento de cross-linking

\begin{tabular}{|c|c|c|c|c|c|}
\hline Estudo* & Olhos & Procedimento & Seguimento & Mudança na acuidade visual & Mudança refrativa \\
\hline Hafezi, 2007(16) & 10 & $\begin{array}{l}\text { CXL para ectasia após } \\
\text { laser in situ keratomileusis }\end{array}$ & 25 meses & $\begin{array}{l}\text { Melhora da AV e cilindro } \\
\text { na maioria dos casos }\end{array}$ & $\begin{array}{l}\text { Diminuição da ceratometria máxima } \\
\text { de 2,0 D ou mais em } 5 \text { olhos }\end{array}$ \\
\hline Raiskup-Wolf, 2008 $8^{(17)}$ & 480 & $\begin{array}{l}\text { CXL para ceratocone } \\
\text { progressivo }\end{array}$ & $\begin{array}{l}\text { Máximo de } 6 \text { anos } \\
\text { e média de } 26 \text { meses }\end{array}$ & $\begin{array}{l}\text { Melhora da AVCC ( } \geq 1 \text { linha) } \\
\text { em } 53 \% \text { dos olhos } \\
\text { no } 1^{\circ} \text { ano e } 57 \% \text { no } 2^{\circ} \text { ano }\end{array}$ & $\begin{array}{l}\text { A ceratometria máxima (K2) diminuiu } \\
\text { de } 2,68 \mathrm{D} \text { no } 1^{\circ} \text { ano, } 2,21 \mathrm{D} \text { no } 2^{\circ} \text { ano } \\
\text { e 4,84 } \text { no } 3^{\circ} \text { ano }(\mathrm{n}=33)\end{array}$ \\
\hline Vinciguerra, 2009(18) & 28 & $\begin{array}{l}\text { CXL para ceratocone } \\
\text { avançado progressivo }\end{array}$ & 1 ano & $\begin{array}{l}\text { A média preop da AVSC e AVCC } \\
\text { foi de } 0,17 \text { e } 0,52 \text { respectivamente; } \\
\text { em } 1 \text { ano, foi de } 0,27 \text { e } 0,72 \text {, ( } p<0,05)\end{array}$ & $\begin{array}{l}\text { A ceratometria média preop } \\
\text { era de } 48,08 \mathrm{D} ; \text { com } 1 \text { ano, } \\
\text { era de } 42,01 \mathrm{D}(\mathrm{p}<0,05)\end{array}$ \\
\hline Caporossi, $2010^{(8)}$ & 44 & CXL para ceratocone & $\begin{array}{l}\text { Média de } \\
52,4 \text { meses }\end{array}$ & $\begin{array}{l}\text { A AVCC melhorou em } \\
1,9 \text { linhas de Snellen, } \\
\text { e a AVSC melhorou de } \\
2,7 \text { linhas de Snellen }\end{array}$ & $\begin{array}{l}\text { A ceratometria média reduziu em } \\
2,0 \text { D, a aberração coma melhorou } \\
\text { em mais de } 85 \% \text { dos casos }\end{array}$ \\
\hline
\end{tabular}

Estudo $^{*}=$ somente primeiro autor; $\mathrm{CXL}=$ cross-linking; $\mathrm{AV}=$ acuidade visual; $\mathrm{D}=$ dioptrias; $\mathrm{AVCC}=$ acuidade visual com correção; $\mathrm{AVSC}=$ acuidade visual sem correção; Preop= pré-operatória 


\section{SEGMENTO DE ANEL CORNEANO INTRAESTROMAL HistóRico}

O conceito de adicionar tecido na periferia da córnea para remodelar sua curvatura anterior foi introduzido por Barraquer em $1949^{(22-23)}$

Em 1987, Fleming et al.(24) descreveram um implante feito de polimetilmetacrilato (PMMA) chamado de anel corneano intraestromal $(\mathrm{ACl})$, baseado em um original conceito desenvolvido por Reynolds ${ }^{(24)}$. As primeiras cirurgias usando os anéis corneanos intraestromais foram realizadas no Brasil em olhos não funcionais por Nosé(25), e nos Estados Unidos por Assil(26), para correção de baixa miopia.

Em 1995, um estudo clínico fase II regulado pelo United States Food and Drug Administration (FDA) teve início usando-se segmentos de anéis corneanos intraestromais $(\mathrm{SACl})$ manufaturados pela Keravision Inc. Em 1999, os SACI foram aprovados pelo FDA para correção de miopia de -1.00 até -3.00 dioptrias (D) recebendo então o nome Intacs. Após falência da Keravision Inc. em 2001, esses anéis passaram a ser representados pela Addition Technology Inc., mantendo-se o nome Intacs.

Em 1986, no Brasil, Ferrara(27) iniciou o uso do SACl para miopias moderadas e altas. Utilizou os segmentos de anéis inicialmente em coelhos albinos, examinados durante 12 meses. Em nenhum caso houve extrusão do implante e sua localização anterior permitiu verificar também um aumento do efeito refrativo do implante ${ }^{(28)}$. Este autor, em 1996, usou o SACI para correção de ceratocone, e em 1999 para correção de astigmatismo irregular após cirurgia refrativa(27).

\section{Mecanismo de AÇÃo}

De acordo com Barraquer ${ }^{(23)}$, quando material é removido da área central da córnea ou adicionado à periferia, um efeito de aplanamento central é alcançado. Ao contrário, quando material é adicionado ao centro ou removido da periferia corneana, há um encurvamento da área central.

Os SACl criam um efeito de encurtamento da lamela corneana, aplanando a área central da córnea. Para correção do astigmatismo, a base de cada segmento pode produzir uma força de tração na superfície, levando a um adicional aplanamento em seu eixo de referência.

A interpretação topográfica de olhos submetidos a inserção do SACI mostra um aplanamento global da córnea, deslocamento do ápice corneano em direção ao centro, preservação da asfericidade corneana e diminuição da irregularidade da superfície(26).

\section{INDICAÇÕES E CONTRAINDICAÇÕES}

As indicações para o uso dos SACI têm aumentado constantemente. Inicialmente indicados para baixa miopia ${ }^{(25-26)}$, frequentemente têm sido mais usados em pacientes com ceratocone (com intolerância ao uso de lente de contato e espessura corneana de pelo menos $400 \mu \mathrm{m}$ na área de inserção), astigmatismo irregular após ceratoplastia penetrante ou lamelar, ectasia corneana após cirurgia refrativa, astigmatismo irregular após ceratotomia radial, degeneração marginal pelúcida, após trauma corneano em superfícies irregulares ${ }^{(29)}$, e mais recentemente associado à técnica do cross-linking corneano ${ }^{(30)}$.

Os SACI não devem ser utilizados em pacientes com ceratocone grave (leitura ceratométrica acima de 70 D), espessura corneana menor de $400 \mu \mathrm{m}$ na área de inserção, opacidades corneanas, hidrópsia, transplantes corneanos descentrados, doença atópica grave, presença de infecção ativa, doença autoimune sistêmica ou localizada, síndrome de erosão corneana recorrente, cicatriz corneana extensa e distrofias corneanas ${ }^{(26-30)}$.

\section{ANÉIS disPoníveIS PARA INSERÇão}

- Intacs Addition Technology, Inc. (Fremont, CA, USA): consiste em um par de segmento semicircular de PMMA, com um compri- mento de arco de $150^{\circ}$, uma base transversa hexagonal e uma seção longitudinal cônica. Cada segmento tem um diâmetro externo de $8,10 \mathrm{~mm}$ e um diâmetro interno de $6,77 \mathrm{~mm}$. O efeito refrativo é modulado por sua espessura, que varia de 250 a $450 \mu \mathrm{m}$. Recentemente, um novo desenho do Intacs (Intacs SK, Addition Technology) foi produzido; tem um diâmetro interno de $6,0 \mathrm{~mm}$ e uma seção transversal oval. Há duas espessuras disponíveis: $400 \mu \mathrm{m}$ (para ceratometrias de 57 a 62 D e cilindro $<5,0$ D) e $450 \mu \mathrm{m}$ (para ceratometrias $>62$ D e cilindro $>5,0 \mathrm{D})^{(31)}$ (Figura $3 \mathrm{~A}$ ).

- Ferrara Ring Segment (Ferrara Ophthalmics, Belo Horizonte, Brasil): esses segmentos são feitos de PMMA Perspex CQ. Estão disponíveis em dois diâmetros, 6,0 mm para miopia até -7,0 D e 5,0 mm para alta miopia. Sua espessura varia de 150 a $350 \mu \mathrm{m}, \mathrm{com}$ mudança a cada $50 \mu \mathrm{m}$. O diâmetro interno e externo é de 4,4 mm e 5,4 mm, respectivamente, para 5,0 $\mathrm{mm}$ de zona óptica e 5,4 $\mathrm{mm}$ e 6,4 mm, respectivamente, para $6,0 \mathrm{~mm}$ de zona ótica. Tem uma base triangular com uma constante de $600 \mu \mathrm{m}$. Os segmentos têm $90^{\circ}, 120^{\circ}$, $150^{\circ}, 160^{\circ}, 210^{\circ}$ ou $240^{\circ}$ de $\operatorname{arco}^{(31)}$ (Figura 3 B).

- Keraring (Mediphacos, Belo Horizonte, Brasil): são feitos de PMMA e caracterizados por uma base triangular de $600 \mu \mathrm{m}$. Os diâmetros apicais são de 5,0 mm ou 6,0 mm e com espessura variando de 150 a $350 \mu \mathrm{m}$, com mudança a cada $50 \mu \mathrm{m}$. Possui comprimento de arco variável $\left(90^{\circ}, 120^{\circ}, 150^{\circ}, 160^{\circ}, 210^{\circ}\right.$ e $\left.240^{\circ}\right)$. Há dois modelos, o SI5 (zona óptica de $5,0 \mathrm{~mm}$ ) e o $\mathrm{SI} 6$ (zona óptica de $5,5 \mathrm{~mm}$ ou $6,0 \mathrm{~mm}$ ) (Figura $3 \mathrm{C}$ ).

- Corneal Ring (Visiontech, Belo Horizonte, Brasil): são feitos de PMMA e caracterizados por uma secção transversal fusiforme. Possui um diâmetro interno de 4,7 mm e a espessura varia de 150 a 350 Mm, com mudança a cada $50 \mu \mathrm{m}$. Tem comprimento de arco de $155^{\circ}$ (comprimento padrão) e $220^{\circ}$ (segmento especial).

\section{Planejamento cirúrgico}

Para a seleção do tipo de segmento de anel a ser inserido e sua posição, o cirurgião deve considerar alguns parâmetros como refração, ceratometria, espessura corneana e a forma do ceratocone de acordo com a topografia. As empresas fabricantes dos segmentos de anéis fornecem nomogramas que auxiliam na escolha desses segmentos.

Há algumas diferenças entre os cirurgiões na escolha da profundidade de implantação do SACl. Relatos na literatura têm mostrado inserção a $66 \%, 70 \%, 75 \%$ e $80 \%$ de profundidade corneana na zona óptica de 5-7 mm. Ertan e Kamburoglu ${ }^{(32)}$ implantaram os segmentos de Intacs em uma profundidade corneana de $70 \%$. Houve extrusão dos segmentos em três olhos em pacientes com ceratocone grave. Coskunseven et al. ${ }^{(33)}$ relataram a inserção do segmento Intacs a uma profundidade corneana de $75 \%$, sem complicações intraoperatórias. É importante seguir o nomograma de cada fabricante a fim de se evitar a utilização de anéis muito espessos (350 $\mu \mathrm{m})$ em córneas finas. Esses nomogramas trazem a informação do limite máximo de espessura do anel a ser inserido levando em consideração a espessura mínima da córnea do paciente no local de inserção do segmento.

\section{Procedimento CirúRgico} local.

O procedimento cirúrgico é realizado sob assepsia e anestesia

\section{1) Técnica mecânica}

O procedimento é centralizado no reflexo corneano da luz do microscópio, onde um marcador circular é usado para criar duas marcas concêntricas na córnea. Usando um bisturi de diamante com a profundidade de inserção escolhida pelo cirurgião, uma incisão corneana radial é criada entre as duas marcações na periferia do cone, no seu eixo mais curvo (alguns cirurgiões fazem uma incisão horizontal). Através do separador corneano estromal na base da 

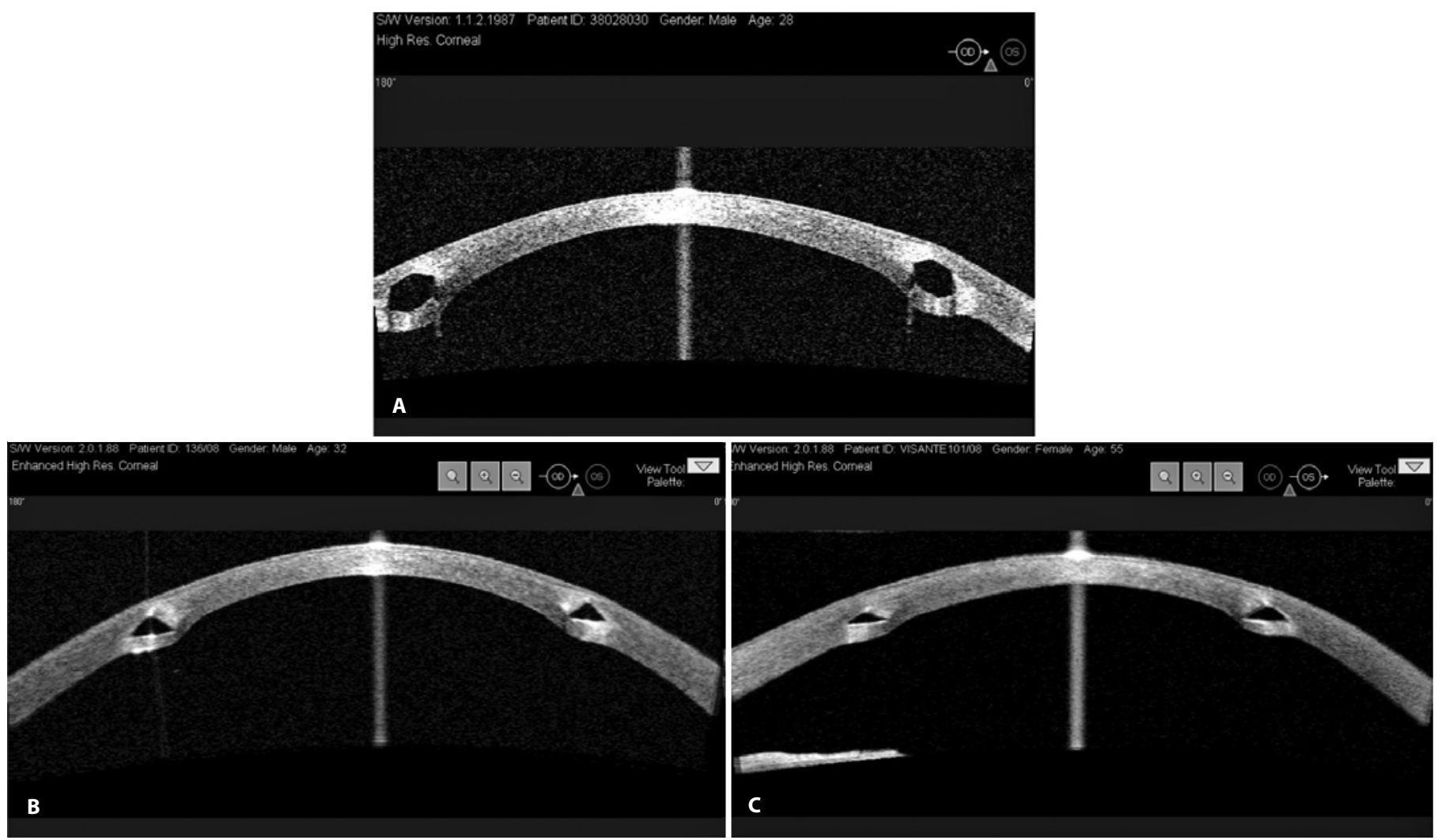

Figura 3. A) Imagem do Visante ${ }^{T M}$ OCT-Intacs; B) Imagem do Visante ${ }^{T M}$ OCT-Ferrara; C) Imagem do Visante ${ }^{T M}$ OCT-Keraring.

incisão, o tecido corneano é divulsionado para a entrada dos anéis. Com os túneis já iniciados, faz-se a confecção de dois túneis concêntricos, no sentido horário e anti-horário, com tunelizadores apropriados. Após, segue-se a inserção dos anéis. Vácuo também pode ser usado para a criação dos túneis. O diâmetro do túnel depende do tipo de anel selecionado. Na maioria dos casos, não há necessidade de sutura. Após a cirurgia, pode ser colocada uma lente de contato terapêutica por 24 a 48h. Medicações pós-operatórias incluem uma combinação de colírio antibiótico e anti-inflamatório hormonal quatro vezes ao dia por 2 semanas, e lágrimas artificiais.

\section{2) Técnica pelo laser de femtosegundo}

O procedimento é centralizado no reflexo corneano da luz do microscópio. Os parâmetros usados para a criação do túnel e incisão são diâmetro interno e externo, comprimento e profundidade da incisão. O túnel é confeccionado pelo laser de femtosegundo (laser infravermelho ultrarrápido $10^{-15}$ de segundo) com um comprimento de onda de $1053 \mathrm{~nm}$. O feixe de laser de $3 \mu \mathrm{m}$ de diâmetro é opticamente focado na profundidade estromal predeterminada no equipamento. Este feixe gera cavidades, microbolhas de dióxido de carbono e vapor de água pelo mecanismo de fotodisrrupção. A interconexão dessas bolhas forma um plano de dissecção. Um anel descartável de sucção é colocado no olho para sua fixação. A criação do túnel intraestromal é completada em 15 segundos, sem manipulação corneana(31). Após, segue-se a colocação do anel.

\section{RESULTADOS DE LITERATURA}

A tabela 2 mostra alguns resultados da literatura em relação à inserção de diferentes tipos de anéis em ceratocone, assim como as duas diferentes técnicas.

\section{Segmentos de anéls corneanos intraestromais para ectasia após} CIRURGIA REFRATIVA E DEGENERAÇÃO MARGINAL PELÚCIDA

Alguns estudos relatam o uso de SACl em pacientes com ectasia após laser in situ keratomileusis (LASIK) e em pacientes com degeneração marginal pelúcida (DMP)(40-41). Kymionis et al. ${ }^{(40)}$ apresentaram resultados de 5 anos de seguimento mostrando estabilidade após inserção do Intacs em pacientes com ectasia pós LASIK.

Em DMP, uma doença progressiva, não inflamatória e periférica da córnea caracterizada por uma faixa de afinamento corneano principalmente inferior, Ertan e Bahadýr(41) implantaram Intacs em 9 olhos com DMP usando o laser de femtosegundo. A acuidade visual não corrigida melhorou em todos os olhos do estudo.

Em um estudo de revisão relacionado ao tratamento de ectasias primárias ou secundárias, Tan et al. mostram que a inserção dos $\mathrm{SACl}$ seria uma alternativa viável à ceratoplastia penetrante convencional, pois melhoram a acuidade e reabilitação visual dos pacientes $^{(42)}$.

A tabela 3 mostra alguns resultados da literatura relacionados a ectasia após cirurgia refrativa ou DMP.

\section{Associação de SEGMENTOS DE ANÉIS CORNEANOS INTRAESTROMAIS E CROSS-LINKING}

Somente a inserção do Intacs pode não impedir a progressão do ceratocone. Alió et al. ${ }^{(50)}$ encontrou um aumento de 1,67 D nos valores de ceratometria média entre 6 meses e 36 meses em uma série de 13 olhos após a implantação do Intacs. Isto pode ser esperado, pois os segmentos de anéis não tratam o problema estrutural de fraqueza do colágeno corneano. Assim, surgiu a idéia da combinação do tratamento cross-linking com a inserção do SACl em pacientes com ceratocone para garantir a estabilidade corneana ${ }^{(31)}$. 
Tabela 2. Resultados de segmentos de anéis intraestromais

\begin{tabular}{|c|c|c|c|c|c|c|}
\hline Estudo* & Olhos & $\begin{array}{l}\text { Intacs }^{\circledR} \\
\text { Keraring }^{\circledR} \\
\text { Ferrara }^{\circledR}\end{array}$ & $\begin{array}{l}\text { Seguimento } \\
\text { médio }\end{array}$ & $\begin{array}{l}\text { Mudança na } \\
\text { acuidade visual }\end{array}$ & $\begin{array}{l}\text { Mudança } \\
\text { refrativa }\end{array}$ & Complicações \\
\hline Coskunseven ${ }^{(33)}$ & 50 & Keraring & 15,6 meses & $\begin{array}{l}\text { Ganho de 1,7 linhas de } \\
\text { Snellen na AVSC }\end{array}$ & $\begin{array}{l}\text { EE médio diminuiu de } \\
-5,62 \mathrm{D} \text { para }-2,50 \mathrm{D}\end{array}$ & $\begin{array}{l}\text { Em } 3 \text { olhos houve migração } \\
\text { do seguimento para a } \\
\text { incisão no } 1^{\circ} \mathrm{PO}\end{array}$ \\
\hline $\operatorname{Ertan}^{(32)}$ & 306 & Intacs & 10 meses & $\begin{array}{l}\text { Ganho de mais de } 4 \text { linhas } \\
\text { de AVSC e AVCC em 15\% e } \\
\text { 10,7\% dos olhos } \\
\text { respectivamente }\end{array}$ & $\begin{array}{l}\text { EE médio diminuiu de } \\
-7,81 \mathrm{D} \text { para }-4,72 \mathrm{D} ; \\
\text { a ceratometria média } \\
\text { diminuiu de } 50,70 \mathrm{D} \\
\text { para 47,91 D }\end{array}$ & $\begin{array}{l}\text { Extrusão do seguimento ocorreu } \\
\text { em } 3 \text { olhos } 6 \text { meses após o } \\
\text { implante do anel }\end{array}$ \\
\hline Torquetti(34) & 35 & Ferrara & 5 anos & $\begin{array}{l}\text { A AVSC melhorou de } \\
0,15 \text { para 0,31 aos } 5 \text { anos, } \\
\text { e a AVCC melhorou de 0,41 } \\
\text { para 0,62 (Snellen) }\end{array}$ & $\begin{array}{l}\text { A ceratometria média } \\
\text { diminuiu de } 51,27 \text { D para } \\
\text { 46,24 D aos } 5 \text { anos }\end{array}$ & $\begin{array}{l}\text { Os autores não relataram } \\
\text { as complicações deste estudo }\end{array}$ \\
\hline Shabayek $^{(35)}$ & 21 & Keraring & 6 meses & $\begin{array}{l}\text { A AVSC melhorou de } \\
0,06 \text { para 0,3; A AVCC } \\
\text { melhorou de 0,54 } \\
\text { para 0,71 (Snellen) }\end{array}$ & $\begin{array}{l}\text { EE médio diminuiu em 2,23 D; } \\
\text { a ceratometria média diminuiu } \\
\text { de } 48,79 \mathrm{D} \text { para } 46,54 \mathrm{D}\end{array}$ & $\begin{array}{l}\text { Ceratite infecciosa localizada } \\
\text { ocorreu em } 1 \text { olho } 1 \text { mês } \\
\text { após a cirurgia; em } 8 \text { olhos, } \\
\text { ocorreu opacificação corneana } \\
\text { superficial no sítio da incisão }\end{array}$ \\
\hline Kwitko(36) & 51 & Ferrara & 13 meses & $\begin{array}{l}\text { A AVSC melhorou em } 44 \text { olhos, } \\
\text { não mudou em } 3 \text { olhos e } \\
\text { piorou em } 3 \text { olhos; } \\
\text { A AVCC melhorou em } 44 \text { olhos, } \\
\text { não mudou em } 1 \text { olho e } \\
\text { piorou em } 6 \text { olhos }\end{array}$ & $\begin{array}{l}\text { EE médio diminuiu de - } 6,08 \mathrm{D} \\
\text { para -3,81 D; o astigmatismo } \\
\text { diminuiu de 3,69 D para 2,20 D; } \\
\text { A ceratometria média diminuiu } \\
\text { de 48,76 D para 43,17 D }\end{array}$ & $\begin{array}{l}\text { Descentração em } 2 \text { olhos; } \\
\text { extrusão do anel em } 10 \text { olhos; } \\
\text { ceratite disciforme em } 1 \text { olho e } \\
\text { ceratite bacteriana em } 1 \text { olho }\end{array}$ \\
\hline $\operatorname{Colin}^{(37)}$ & 10 & Intacs & 12 meses & $\begin{array}{l}\text { A AVSC melhorou em } \\
\text { média de } 20 / 200 \text { para 20/50 } \\
\text { no PO de } 12 \text { meses; } \\
\text { A AVCC melhorou em média } \\
\text { de } 20 / 50 \text { para } 20 / 32 \text { no } \\
\text { PO de } 12 \text { meses }\end{array}$ & $\begin{array}{l}\text { O astigmatismo reduziu em média } \\
\text { de } 4 \text { D para 1,3 D no PO } 12 \text { meses; } \\
\text { Redução da ceratometria } \\
\text { média mínima de } 48 \text { D para } \\
\text { 44,40 D aos } 12 \text { meses, e redução da } \\
\text { ceratometria média máxima de } \\
\text { 53,2 D para 48,6 D aos } 12 \text { meses }\end{array}$ & $\begin{array}{l}\text { Sem complicações } \\
\text { intraoperatórias nesta } \\
\text { série de casos }\end{array}$ \\
\hline Bryan $^{(38)}$ & 13 & Intacs & 6 meses & $\begin{array}{l}\text { Houve média de } \\
\text { ganho de } 1 \text { linha tanto } \\
\text { na AVSC como também } \\
\text { na AVCC, do pré-operatório } \\
\text { até } 6 \text { meses de seguimento }\end{array}$ & $\begin{array}{l}\text { O EE não mudou dos valores } \\
\text { pré-operatórios até } \\
\text { os } 6 \text { meses de seguimento; } \\
\text { Houve uma redução em } 2,77 \text { D } \\
\text { no astigmatismo, do pré-operatório } \\
\text { para } 6 \text { meses de seguimento }\end{array}$ & Sem complicações descritas \\
\hline Colin $^{(39)}$ & 10 & Intacs & 10,6 meses & $\begin{array}{l}\text { A AVSC melhorou de 0,12 } \\
\text { para 0,3 (Snellen) aos } 6 \text { meses; } \\
\text { A AVCC melhorou de 0,38 } \\
\text { para } 0,63 \text { aos } 6 \text { meses } \\
\text { de seguimento }\end{array}$ & $\begin{array}{l}\text { O EE reduziu de -5,13 D para } \\
-3,01 \text { D aos } 6 \text { meses; } \\
\text { A ceratometria média reduziu } \\
\text { de } 51,73 \text { D para } 46,88 \text { D } \\
\text { aos } 6 \text { meses de seguimento }\end{array}$ & $\begin{array}{l}\text { Sem complicação } \\
\text { intraoperatória } \\
\text { Extrusão do anel em } 1 \text { olho } \\
\text { após } 2 \text { meses da cirurgia }\end{array}$ \\
\hline
\end{tabular}

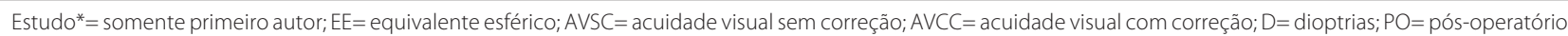

Há ainda poucos estudos associando as duas técnicas descritos até o momento:

Chan et al. ${ }^{(51)}$ mostraram uma adicional razão para a combinação das duas terapias. Em um estudo com 25 olhos, os autores encontraram um maior efeito de aplanamento após a inserção do Intacs no grupo de pacientes que foram submetidos ao CXL (após Intacs). Houve redução estatisticamente significante maior nos valores de cilindro e ceratometria no grupo (CXL+Intacs) em relação ao grupo Intacs somente.

Coskunseven et al. ${ }^{(30)}$ compararam duas sequências de tratamento, (CXL+Keraring) e (Keraring $+C X L)$. A acuidade visual média não corrigida e corrigida melhoraram em ambos os grupos. Os valores médios de equivalente esférico, cilindro e ceratometria também diminuíram em ambos os grupos. Os autores concluiram que de maneira geral, houve uma maior melhora na acuidade visual corrigida, equivalente esférico e ceratometria média no grupo (Keraring $+\mathrm{CXL})$.

Em um estudo onde 25 olhos de pacientes com ceratocone foram submetidos à inserção do Intacs e após, CXL transepitelial (sem remoção de epitélio), Ertan et al. ${ }^{(52)}$ verificaram que após o
CXL, houve uma melhora de 1,2 linhas de Snellen na acuidade visual não corrigida e 0,36 linhas de Snellen na acuidade visual corrigida. Houve adicional diminuição nos valores de grau esférico, cilíndrico, ceratometria média e mais curva de 0,5 D, 0,15 D, 0,35 D e 0,76 D respectivamente após o tratamento do CXL. Os autores concluiram que a implantação do Intacs seguida do CXL transepitelial foi efetiva em olhos com ceratocone.

Há algumas possíveis razões para o maior efeito com a adição do tratamento cross-linking. Inicialmente, ele pode funcionar como um simples efeito aditivo, como mostrou Wollensak et al. ${ }^{(4)} \mathrm{em}$ seu estudo original. Eles encontraram uma redução média da ceratometria máxima de 2,01 D somente com o cross-linking em 70\% dos olhos estudados.

\section{COMPLICAÇÕES}

A técnica mecânica tradicional de criação do túnel intraestromal pode levar às seguintes complicações ${ }^{(31,53)}$ : defeito epitelial no sítio da incisão, perfuração anterior e posterior durante a criação do túnel, extensão da incisão em direção ao eixo visual ou limbo, 
Tabela 3. Resultados de segmentos de anéis intraestromais pós LASIK/PRK ou DMP

\begin{tabular}{|c|c|c|c|c|c|c|}
\hline Estudo* & Olhos & $\begin{array}{l}\text { Intacs }^{\circledR} \\
\text { Keraring }^{\circledR} \\
\text { Ferrara }^{\circledR}\end{array}$ & $\begin{array}{l}\text { Seguimento } \\
\text { médio }\end{array}$ & $\begin{array}{l}\text { Mudança na } \\
\text { acuidade visual }\end{array}$ & $\begin{array}{l}\text { Mudança } \\
\text { refrativa }\end{array}$ & Complicações \\
\hline Mularoni(43) & 8 & Intacs/DMP & 24 meses & $\begin{array}{l}\text { A AVSC melhorou de 20/325 } \\
\text { para } 20 / 50 \text { aos } 12 \text { meses; } \\
\text { A AVCC melhorou de } 20 / 45 \\
\text { para } 20 / 30 \text { aos } 12 \text { meses }\end{array}$ & $\begin{array}{l}\text { EE médio diminuiu } \\
\text { de }-4,75 \text { D para }-1,36 \text { D; } \\
\text { Ocilindro médio diminuiu } \\
\text { de }-6,31 \text { D para -1,72 D }\end{array}$ & Sem complicações \\
\hline Piñero ${ }^{(44)}$ & 21 & $\begin{array}{c}\text { Intacs e } \\
\text { Keraring/DMP }\end{array}$ & 6 meses & $\begin{array}{l}\text { A AVSC não mudou ao } \\
\text { longo de } 6 \text { meses; } \\
\text { A AVCC melhorou em } \\
44,44 \% \text { dos olhos, } \\
\text { com ganho de } 2 \text { linhas }\end{array}$ & $\begin{array}{l}\text { EE médio diminuiu } \\
\text { de -3,84 D para - } 0,87 \mathrm{D} ; \\
\text { O cilindro médio reduziu de } \\
\text {-5,36 D para -3,21 D; } \\
\text { Redução da ceratometria } \\
\text { média de 44,95 D para 43,19 D }\end{array}$ & Sem complicações \\
\hline $\operatorname{Ertan}^{(45)}$ & 1 & Intacs/DMP & 3 meses & $\begin{array}{l}\text { A AVSC melhorou } \\
\text { de } 0,05 \text { para } 0,15 \text {; A AVCC } \\
\text { melhorou de } 0,15 \text { para 0,4 }\end{array}$ & $\begin{array}{l}\text { A refração melhorou de } \\
-4,50 \times 180^{\circ} \text { para }-2,50 \times 90^{\circ}\end{array}$ & Sem complicações \\
\hline Pokroy ${ }^{(46)}$ & 5 & Intacs/LASIK & 9 meses & $\begin{array}{l}\text { Houve melhora de } \\
\text { linhas de visão em } \\
\text { todos os casos }\end{array}$ & $\begin{array}{l}\text { O EE médio melhorou de } \\
-1,60 \text { D para }-0,80 \text { D }\end{array}$ & Sem complicações \\
\hline Kymionis ${ }^{(47)}$ & 8 & Intacs/LASIK & 5 anos & $\begin{array}{l}\text { A AVSC melhorou de } 20 / 100 \\
\text { para } 20 / 40 \text { ou melhor em } 6 \text { olhos; } \\
\text { Houve ganho de } 1 \text { ou } \\
2 \text { linhas na AVCC em } 6 \text { olhos }\end{array}$ & $\begin{array}{l}\text { O EE médio melhorou de } \\
-5,47 \text { D para }-2,56 \text { D; } \\
\text { A ceratometria média melhorou } \\
\text { de } 41,29 \text { D para } 38,04 \text { D, com } \\
\text { redução média de 3,68 D }\end{array}$ & Sem complicações \\
\hline Lovisolo ${ }^{(48)}$ & 4 & $\begin{array}{l}\text { Intacs / Anel } \\
\text { de Ferrara } \\
\text { LASIK/ PRK }\end{array}$ & 3 meses & $\begin{array}{l}\text { Melhora significante na } \\
\text { AVSC e AVCC }\end{array}$ & $\begin{array}{l}\text { Regularização da superfície } \\
\text { corneana com aumento } \\
\text { de sua uniformidade }\end{array}$ & Sem complicações \\
\hline Kymionis ${ }^{(49)}$ & 10 & Intacs/LASIK & 15 meses & $\begin{array}{l}\text { A AVSC melhorou de } 20 / 100 \\
\text { para } 20 / 40 \text { ou melhor em } 9 \text { olhos; } \\
\text { A AVCC melhorou em } 7 \text { olhos, } \\
\text { com ganho de } 1 \text { a } 2 \text { linhas }\end{array}$ & $\begin{array}{l}\text { O EE médio melhorou de } \\
-4,81 \text { D para }-0,96 \text { D; } \\
\text { A ceratometria média reduziu de } \\
40,21 \text { D para } 37,14 \text { D, } \\
\text { com redução média de } 3,07 \mathrm{D}\end{array}$ & Sem complicações \\
\hline Alió(50) & 3 & Intacs/LASIK & 8 meses & $\begin{array}{l}\text { A AVSC foi para } 20 / 40 \text { em } \\
2 \text { olhos e } 20 / 50 \text { em } 1 \text { olho; } \\
\text { A AVCC foi para } 20 / 40 \text { em } \\
2 \text { olhos e } 20 / 32 \text { em } 1 \text { olho }\end{array}$ & $\begin{array}{l}\text { A ceratometria máxima reduziu de } \\
33,60 \text { para } 28,90 \mathrm{D} \text { em } 1 \text { olho, } \\
38,10 \text { para } 32,90 \mathrm{D} \text { no segundo olho, } \\
\text { e de } 37,80 \text { para } 33,90 \mathrm{D} \text { no terceiro olho }\end{array}$ & Sem complicações \\
\hline
\end{tabular}

Estudo* $^{*}$ somente primeiro autor; $E \mathrm{E}=$ equivalente esférico; $\mathrm{AVSC}=$ acuidade visual sem correção; $\mathrm{AVCC}=$ acuidade visual com correção; $\mathrm{D}=$ dioptrias

superficialização dos segmentos de anéis, ceratite infecciosa, intervalo incisional persistente, descentração do anel, afinamento estromal, edema corneano estromal ao redor da incisão ou do anel, extrusão do segmento e migração, hipocorreção ou hipercorreção do erro refrativo, depósitos intraestromais e "glare".

O laser de femtosegundo oferece várias vantagens que poderiam reduzir estas complicações devido a uma localização mais precisa do túnel e de suas dimensões, no que se refere à profundidade, diâmetro e largura. Entretanto, há relatos de complicações. Coskunseven et al. ${ }^{(54)}$ analisando 850 olhos com ceratocone que foram submetidos à inserção do Keraring pelo laser de femtosegundo, observaram que a criação incompleta do túnel foi a complicação intraoperatória mais frequente, e migração do segmento de anel a complicação pós-operatória mais frequente. A razão geral de complicações foi de 5,7\% (49 casos).

Em uma revisão retrospectiva de 74 olhos de 45 pacientes ${ }^{(55)}$, Intacs foi implantado em 4 olhos, porém explantado em 3 olhos devido a intenso desconforto ou baixa permanente de acuidade visual. Subsequentemente estes olhos foram submetidos a transplante penetrante de córnea com boa evolução.

\section{CONCLUSÃO}

O cross-linking é um método que está se mostrando promissor para o tratamento de ceratocone. Sua principal indicação é para pacientes que apresentam ceratocone com progressão documentada. Os segmentos de anéis intracorneanos visam à regularização da superfície corneana pelo seu efeito de aplanamento. A associação das técnicas de inserção do segmento de anel e cross-linking podem proporcionar melhor efeito no tratamento das ectasias corneanas.

\section{REFERÊNCIAS}

1. Andreassen $\Pi$ T, Simonsen $\mathrm{AH}, \mathrm{Oxlund} \mathrm{H}$. Biomechanical properties of keratoconus and normal corneas. Exp Eye Res.1980;31(4):435-41.

2. Wollensak J, Ihme A, Seiler T. Neue Befunde bei Keratoconus. Fortschr Ophthalmol. 1987;84:28-32.

3. Spoerl E, Huhle M, Seiler T. Induction of cross-links in corneal tissue. Exp Eye Res. 1998; 66(1):97-103.

4. Wollensak G, Spoerl E, Seiler T. Riboflavin/ultraviolet-A-induced collagen crosslinking for the treatment of keratoconus. Am J Ophthalmol. 2003;135(5):620-7.

5. Wollensak G. Crosslinking treatment of progressive keratoconus: new hope. Curr Opin Ophthalmol. 2006;17(4):356-60.

6. Spoerl E, Mrochen M, Sliney D, Trokel S, Seiler T. Safety of UVA-riboflavin cross-linking of the cornea. Cornea. 2007;26(4):385-9.

7. Bottós KM, Dreyfuss JL, Regatieri CV, Lima-Filho AA, Schor P, Nader HB, Chamon W. Immunofluorescence confocal microscopy of porcine corneas following collagen crosslinking treatment with riboflavin and ultraviolet A. J Refract Surg. 2008; 24(7):S715-9.

8. Caporossi A, Mazzotta C, Baiocchi S, Caporossi T. Long-term results of riboflavin ultraviolet A corneal collagen cross-linking for keratoconus in Italy: the Siena eye cross study. Am J Ophthalmol. 2010;149(4):585-93. Comment in: Am J Ophthalmol. 2010; 150(4):588; author reply 588-9.

9. Wollensak G, Spoerl E, Seiler T. Stress-strain measurements of human and porcine corneas after riboflavin-ultraviolet-A-induced cross-linking. J Cataract Refract Surg. 2003;29(9): 1780-5.

10. Spoerl E, Wollensak G, Dittert DD, Seiler T. Thermomechanical behavior of collagen-crosslinked porcine cornea. Ophthalmologica. 2004;218(2):136-40. 
11. Wollensak G, Wilsch M, Spoerl E, Seiler T. Collagen fiber diameter in the rabbit cornea after collagen crosslinking by riboflavin/UVA. Cornea. 2004;23(5):503-7. Comment in: Cornea. 2004;23(5):432.

12. Mazzotta C, Traversi C, Baiocchi S, Caporossi O, Bovone C, Sparano MC, et al. Corneal healing after riboflavin ultraviolet-A collagen cross-linking determined by confocal laser scanning microscopy in vivo: early and late modifications. Am J Ophthalmol. 2008; 146(4):527-33.

13. Wollensak G, Spoerl E, Wilsch M, Seiler T. Keratocyte apoptosis after corneal collagen crosslinking using riboflavin/UVA treatment. Cornea. 2004;23(1):43-9.

14. Kohlhaas M, Spoerl E, Schilde T, Unger G, Wittig C, Pillunat LE. Biomechanical evidence of the distribution of cross-links in corneas treated with riboflavin and ultraviolet A light. J Cataract Refract Surg. 2006:32(2):279-83

15. Spoerl E, Wollensak G, Seiler T. Increased resistance of crosslinked cornea against enzymatic digestion. Curr Eye Res. 2004;29(1):35-40

16. Hafezi F, Kanellopoulos J, Wiltfang R, Seiler T. Corneal collagen crosslinking with riboflavin and ultraviolet A to treat induced keratectasia after laser in situ keratomileusis. J Cataract Refract Surg. 2007;33(12):2035-40. Comment in: J Cataract Refract Surg. 2008;34(6):879; author reply 879 .

17. Raiskup-Wolf F, Hoyer A, Spoerl E, Pillunat LE. Collagen crosslinking with riboflavin and ultraviolet-A light in keratoconus: long-term results. J Cataract Refract Surg. 2008; 34(5):796-801.

18. Vinciguerra P, Albè E, Trazza S, Rosetta P, Vinciguerra R, Seiler T, Epstein D. Refractive, topographic, tomographic, and aberrometric analysis of keratoconic eyes undergoing corneal cross-linking. Ophthalmology. 2009;116(3):369-78. Comment in: Ophthalmology. 2009;116(10):2036-7; author reply 2037-8.

19. Wollensak G, Spoerl E, Wilsch M, Seiler T. Endothelial cell damage after riboflavinultraviolet-A treatment in the rabbit. J Cataract Refract Surg. 2003;29(9):1786-90.

20. Koller T, Mrochen M, Seiler T. Complication and failure rates after corneal crosslinking. J Cataract Refract Surg. 2009;35(8):1358-62. Comment in: J Cataract Refract Surg. 2010; 36(1):185; author reply 186.

21. Kymionis GD, Bouzoukis DI, Diakonis VF, Portaliou DM, Pallikaris Al, Yoo SH. Diffuse lamellar keratitis after corneal crosslinking in a patient with post-laser in situ keratomileusis corneal ectasia. J Cataract Refract Surg. 2007:33(12):2135-7.

22. Barraquer II. Queratoplastia refractiva. Est Inform Oftalmol. 1949;2:10-30.

23. Barraquer Jl. Modification of refraction by means of intracorneal inclusions. Int Opthalmol Clin. 1966;6(1):53-78.

24. Fleming JF, Reynolds AE, Kilmer L, Burris TE, Abott RL, Schanzlin DJ. The intrastromal corneal ring: two cases in rabbits. J Refract Surg. 1987;3:227-32.

25. Nosé W, Neves RA, Schanzlin DJ, Belfort Júnior R. Intrastromal corneal ring-one-year results of first implants in humans: a preliminary nonfunctional eye study. Refract Corneal Surg. 1993;9(6):452-8.

26. Assil KK, Barrett AM, Fouraker BD, Schanzlin DJ. One-year results of the intrastromal corneal ring in nonfunctional human eyes. Intrastromal Corneal Ring Study Group. Arch Ophthalmol. 1995;113(2):159-67.

27. Silva FBD, Alves EAF, Cunha PFA. Utilização do Anel de Ferrara na estabilização e correção da ectasia corneana pós PRK. Arq Bras Oftalmol. 2000;63(3):215-8.

28. Cunha PFA. Técnica cirúrgica para correção de miopia. Anel corneano intra-estromal. Rev Bras Oftalmol. 1995;54(8):578-88.

29. Ferrara P, Bicalho F. Anel de Ferrara. Rio de Janeiro: Cultura Médica; 2006. p.53-127.

30. Coskunseven E, Jankov MR 2nd, Hafezi F, Atun S, Arslan E, Kymionis GD. Effect of treatment sequence in combined intrastromal corneal rings and corneal collagen crosslinking for keratoconus. J Cataract Refract Surg.2009:35(12):2084-91.

31. Ertan A, Colin J. Intracorneal rings for keratoconus and keratectasia. J Cataract Refract Surg. 2007;33(7):1303-14.

32. Ertan A, Kamburoðlu G. Intacs implantation using a femtosecond laser for management of keratoconus: Comparison of 306 cases in different stages. J Cataract Refract Surg. 2008;34(9):1521-6.
33. Coskunseven E, Kymionis GD, Tsiklis NS, Atun S, Arslan E, Jankov MR, Pallikaris IG. One-year results of intrastromal corneal ring segment implantation (KeraRing) using femtosecond laser in patients with keratoconus. Am J Ophthalmol. 2008;145(5):775-9.

34. Torquetti L, Berbel RF, Ferrara P. Long-term follow-up of intrastromal corneal ring segments in keratoconus. J Cataract Refract Surg. 2009;35(10):1768-73.

35. Shabayek MH, Alió JL. Intrastromal corneal ring segment implantation by femtosecond laser for keratoconus correction. Ophthalmology. 2007;114(9):1643-52.

36. Kwitko S, Severo NS. Ferrara intracorneal ring segments for keratoconus. J Cataract Refract Surg. 2004;30(4):812-20

37. Colin J, Cochener B, Savary G, Malet F, Holmes-Higgin D. INTACS inserts for treating keratoconus: one-year results. Ophthalmology. 2001;108(8):1409-14.

38. Tan BU, Purcell TL, Torres LF, Schanzlin DJ. New surgical approaches to the management of keratoconus and post-LASIK ectasia. Trans Am Ophthalmol Soc. 2006;104:212-20.

39. Colin J, Cochener B, Savary G, Malet F. Correcting keratoconus with intracorneal rings. J Cataract Refract Surg. 2000;26(8):1117-22. Comment in: J Cataract Refract Surg. 2000;26(8): 1099-100. J Cataract Refract Surg. 2001:27(3):341.

40. Kymionis GD, Tsiklis NS, Pallikaris Al, Kounis G, Diakonis VF, Astyrakakis N, Siganos CS. Long-term follow-up of intacs for post-LASIK corneal ectasia. Ophthalmology. 2006; 113(11):1909-17.

41. Ertan A, Bahadir, M. Intrastromal ring segment insertion using a femtosecond laser to correct pellucid marginal corneal degeneration. J Cataract Refract Surg. 2006;32(10): $1710-6$.

42. Tan DT, Por YM. Current treatment options for corneal ectasia. Curr Opin Ophthalmol. 2007:18(4):284-9. Review.

43. Mularoni A, Torreggiani A, di Biase A, Laffi GL, Tassinari G. Conservative treatment of early and moderate pellucid marginal degeneration: a new refractive approach with intracorneal rings. Ophthalmology. 2005;112(4):660-6

44. Piñero DP, Alió JL, Morbelli H, Uceda-Montanes A, El Kady B, Coskunseven E, Pascual I. Refractive and corneal aberrometric changes after intracorneal ring implantation in corneas with pellucid marginal degeneration. Ophthalmology. 2009;116(9):1656-64.

45. Ertan A, Bahadir M. Management of superior pellucid marginal degeneration with a single intracorneal ring segment using femtosecond laser. J Refract Surg. 2007;23(2):205-8.

46. Pokroy R, Levinger S, Hirsh A. Single intacs segment for post-laser in situ keratomileusis keratectasia. J Cataract Refract Surg. 2004:30(8):1685-95.

47. Lovisolo CF, Fleming JF. Intracorneal ring segments for iatrogenic keratectasia after laser in situ keratomileusis or photorefractive keratectomy. J Refract Surg. 2002;18(5):535-41.

48. Kymionis GD, Siganos CS, Kounis G, Astyrakakis N, Kalyvianaki MI, Pallikaris IG. Management of post-LASIK corneal ectasia with Intacs inserts: one-year results. Arch Ophthalmol. 2003;121(3):322-6.

49. Alió J, Salem T, Artola A, Osman A. Intracorneal rings to correct corneal ectasia after laser in situ keratomileusis. J Cataract Refract Surg. 2002;28(9):1568-74.

50. Alió JL, Shabayek MH, Artola A. Intracorneal ring segments for keratoconus correction: long-term follow-up. J Cataract Refract Surg. 2006;32(6):978-85.

51. Chan CC, Sharma M, Wachler BS. Effect of inferior-segment Intacs with and without C3-P on keratoconus. J Cataract Refract Surg. 2007:33(1):75-80. Comment in: J Cataract Refract Surg. 2007;33(8):1352; author reply 1352-3.

52. Ertan A, Karacal H, Kamburoðlu G. Refractive and topographic results of transepithelia cross-linking treatment in eyes with intacs. Cornea. 2009;28(7):719-23.

53. Miranda D, Sartori M, Francesconi C, Allemann N, Ferrara P, Campos M. Ferrara intrastromal corneal ring segments for severe keratoconus. J Refract Surg. 2003;19(6):645-53.

54. Coskunseven E, Kymionis GD, Tsiklis NS, Atun S, Arslan E, Siganos CS, et al. Complications of intrastromal corneal ring segment implantation using a femtosecond laser for channel creation: a survey of 850 eyes with keratoconus. Acta Ophthalmol. 2009 Aug 14 [Epub ahead of print].

55. Woodward MA, Randleman JB, Russel B, Lynn MJ, Ward MA, Stulting RD. Visual rehabilitation and outcomes for ectasia after corneal refractive surgery. J Cataract Refract Surg. 2008;34(3):383-8 